OPEN ACCESS

Edited by:

Ekaterina Shelest

Hans-Knöll-Institute, Germany

Reviewed by:

Julio Vera González,

University Hospital Erlangen, Germany

Nils Blüthgen,

Charité-Universitätsmedizin

Berlin, Germany

*Correspondence:

Hauke Busch

h.busch@dkfz.de;

Melanie Boerries

m.boerries@dkfz.de

${ }^{\dagger}$ Present Address:

Steffen Knauer

Cold Spring Harbor Laboratory, Cold

Spring Harbor, NY, USA

${ }^{\ddagger}$ These authors are co-first authors.

$\S$ These authors are co-last authors.

Specialty section:

This article was submitted to Bioinformatics and Computational

Biology,

a section of the journa

Frontiers in Genetics

Received: 20 November 2015

Accepted: 14 March 2016

Published: 14 April 2016

Citation:

Offermann B, Knauer S, Singh A, Fernández-Cachón ML, Klose M, Kowar S, Busch H and Boerries M (2016) Boolean Modeling Reveals the Necessity of Transcriptional Regulation for Bistability in PC12 Cell

Differentiation. Front. Genet. 7:44. doi: 10.3389/fgene.2016.00044

\section{Boolean Modeling Reveals the Necessity of Transcriptional Regulation for Bistability in PC12 Cell Differentiation}

\author{
Barbara Offermann ${ }^{1 \neq}$, Steffen Knauer ${ }^{1+\neq}$, Amit Singh ${ }^{\ddagger}$, María L. Fernández-Cachón ${ }^{1}$, \\ Martin Klose ${ }^{1}$, Silke Kowar ${ }^{1}$, Hauke Busch ${ }^{1,2,3 * \S}$ and Melanie Boerries ${ }^{1,2,3 * \S}$ \\ 'Systems Biology of the Cellular Microenvironment Group, Institute of Molecular Medicine and Cell Research, \\ Albert-Ludwigs-University Freiburg, Freiburg, Germany, ${ }^{2}$ German Cancer Consortium, Freiburg, Germany, ${ }^{3}$ German Cancer \\ Research Center, Heidelberg, Germany
}

The nerve growth factor NGF has been shown to cause cell fate decisions toward either differentiation or proliferation depending on the relative activity of downstream pERK, pAKT, or pJNK signaling. However, how these protein signals are translated into and fed back from transcriptional activity to complete cellular differentiation over a time span of hours to days is still an open question. Comparing the time-resolved transcriptome response of NGF- or EGF-stimulated PC12 cells over $24 \mathrm{~h}$ in combination with protein and phenotype data we inferred a dynamic Boolean model capturing the temporal sequence of protein signaling, transcriptional response and subsequent autocrine feedback. Network topology was optimized by fitting the model to time-resolved transcriptome data under MEK, PI3K, or JNK inhibition. The integrated model confirmed the parallel use of MAPK/ERK, PI3K/AKT, and JNK/JUN for PC12 cell differentiation. Redundancy of cell signaling is demonstrated from the inhibition of the different MAPK pathways. As suggested in silico and confirmed in vitro, differentiation was substantially suppressed under JNK inhibition, yet delayed only under MEK/ERK inhibition. Most importantly, we found that positive transcriptional feedback induces bistability in the cell fate switch. De novo gene expression was necessary to activate autocrine feedback that caused Urokinase-Type Plasminogen Activator (UPA) Receptor signaling to perpetuate the MAPK activity, finally resulting in the expression of late, differentiation related genes. Thus, the cellular decision toward differentiation depends on the establishment of a transcriptome-induced positive feedback between protein signaling and gene expression thereby constituting a robust control between proliferation and differentiation.

Keywords: PC12 cells, Boolean modeling, NGF signaling, EGF signaling, bistability

\section{INTRODUCTION}

The rat pheochromocytoma cells PC12 are a long established in vitro model to study neuronal differentiation, proliferation and survival (Greene and Tischler, 1976; Burstein et al., 1982; Cowley et al., 1994). After stimulation with the nerve growth factor (NGF), a small, secreted protein from the neurotrophin family, PC12 cells differentiate into sympathetic neuron-like cells, which is 
morphologically marked by neurite outgrowth over a time course of up to 6 days (Levi-Montalcini, 1987; Chao, 1992; Fiore et al., 2009; Weber et al., 2013). NGF binds with high affinity to the TrkA receptor (tyrosine kinase receptor A), thereby activating several downstream protein signaling pathways including primarily the protein kinase C/phospholipase C (PKC/PLC), the phosphoinositide 3-kinase/protein kinase B (PI3K/AKT) and the mitogen-activated protein kinase/extracellular signalregulated kinase (MAPK/ERK) pathways (Kaplan et al., 1991; Jing et al., 1992; Vaudry et al., 2002). Beyond these immediate downstream pathways, further studies showed the involvement of Interleukin 6 (IL6), Urokinase plasminogen activator (uPA) and Tumor Necrosis Factor Receptor Superfamily Member 12A (TNFRSF12A) in PC12 cell differentiation (Marshall, 1995; Wu and Bradshaw, 1996; Leppä et al., 1998; Xing et al., 1998; FariasEisner et al., 2000, 2001; Vaudry et al., 2002; Tanabe et al., 2003). Sustained ERK activation is seen as necessary and sufficient for the successful PC12 cell differentiation under NGF stimulation (Avraham and Yarden, 2011; Chen et al., 2012), whereas transient ERK activation upon epidermal growth factor (EGF) stimulation results in proliferation (Gotoh et al., 1990; Qui and Green, 1992; Marshall, 1995; Vaudry et al., 2002). In fact, selective pathway inhibition or other external stimuli that modulate the duration of ERK activation likewise determine the cellular decision between proliferation and differentiation (Dikic et al., 1994; Vaudry et al., 2002; Santos et al., 2007). Consequently, the MAPK signaling network, as the key pathway in the cellular response, has been studied thoroughly in vitro and in silico (Sasagawa et al., 2005; von Kriegsheim et al., 2009; Saito et al., 2013). Interestingly, both EGF and NGF provoke a similar transcriptional program within the first hour. Therefore, differences in cellular signaling must be due (i) to differential regulation of multiple downstream pathways and (ii) late gene response programs $(>1 \mathrm{~h})$ that feed back into the protein signaling cascade. As an example for pathway crosstalk, both, the MAPK/ERK and c-Jun N-terminal kinase (JNK) pathways regulate c-Jun activity and are necessary for PC12 cell differentiation (Leppä et al., 1998; Waetzig and Herdegen, 2003; Marek et al., 2004), while uPA receptor (uPAR) signaling, as a result of transcriptional AP1 (Activator Protein-1) regulation, is necessary for differentiation of unprimed PC12 cells (Farias-Eisner et al., 2000; Mullenbrock et al., 2011).

In the present study, we combined time-resolved transcriptome analysis of EGF and NGF stimulated PC12 cells up to $24 \mathrm{~h}$ with inhibition of MAPK/ERK, JNK/JUN, and PI3K/AKT signaling, to develop a Boolean Model of PC12 cell differentiation that combines protein signaling, gene regulation and autocrine feedback. The Boolean approach allows to derive important predictions without detailed quantitative kinetic data and parameters over different time scales (Singh et al., 2012). Protein signaling comprised MAPK/ERK, JNK/JUN, and PI3K/AKT pathways. Based on the upstream transcription factor analysis and transcriptional regulation of $M m p 10$ (Matrix Metallopeptidase 10), Serpine1 (Serpin Peptidase Inhibitor, Clade E, Member) and Itgal (Integrin, Alpha 1), we further included an autocrine feedback via uPAR signaling. The model topology was trained on the transcriptional response after pathway inhibition. Inhibition of JNK completely blocked
PC12 cell differentiation and long-term expression of target transcription factors (TFs), such as various Kruppel-like factors (Klf2, 4, 6 and 10), Maff (V-Maf Avian Musculoaponeurotic Fibrosarcoma Oncogene Homolog F) and AP1. Interestingly, inhibition of MEK (mitogen-activated protein kinase kinase), blocking the phosphorylation of ERK, slowed down, but not completely abolished cell differentiation. Neurite quantification over 6 days confirmed a late and reduced, but significant PC12 differentiation, which hinted at alternative pathway usage through JNK. Inhibition of the PI3K/AKT pathway, which is involved in cell proliferation (Chen et al., 2012), even increased the neuronal morphology and neurite outgrowth.

In conclusion, our Boolean modeling approach shows the complex interplay of protein signaling, transcription factor activity and gene regulatory feedback in the decision and perpetuation of PC12 cell differentiation after NGF stimulation.

\section{MATERIALS AND METHODS}

\subsection{Cell Culture and Stimulation}

PC12 cells were obtained from ATCC (American Type Culture Collection, UK) and were cultured at $37^{\circ} \mathrm{C}$ at $5 \% \mathrm{CO}_{2}$ in RPMI 1640 medium, supplemented with 10\% Horse Serum, 5\% Fetal Bovine Serum, 1\% penicillin/streptomycin (PAN Biotech, Germany) and $1 \%$ glutamine (PAN Biotech, Germany). For cell stimulation, 500,000 cells/well were seeded on collagen coated 6 well plates (Corning, NY, USA). The following day, cells were stimulated with $50 \mathrm{ng} / \mathrm{ml}$ rat nerve growth factor (NGF; Promega, Madison, WI, USA) or $75 \mathrm{ng} / \mathrm{ml}$ epidermal growth factor (EGF; R\&D Systems; Wiesbaden, Germany) for the corresponding times. For the pathway inhibition experiments, the following inhibitors were used and added 60 min before NGF was added, mitogen-activated protein inhibitor at a concentration of $20 \mu \mathrm{M}$ (MEKi; U0126 from Promega, Madison, WI, USA), phosphoinositide 3-kinase inhibitor at a concentration of $40 \mu \mathrm{M}$ (PI3Ki; LY-294002 from Enzo Life Sciences, New York, USA) and c-Jun N-terminal kinase inhibitor at a concentration of $20 \mu \mathrm{M}$ (JNKi; SP600125 from SigmaAldrich, St. Louis, USA). The inhibitors were dissolved in DMSO and were further diluted in cell culture medium at their working concentration. Control cells were treated with DMSO at the same concentration that was present in the cells with inhibitor treatment.

\subsection{RNA Isolation and Quantitative Real Time PCR (qRT-PCR)}

Total RNA was isolated from 500,000 cells per timepoint according to the manufacturer's protocol (Universal RNA Purification Kit, Roboklon, Germany). RNA integrity was measured using an Agilent Bioanalyzer-2000 (Agilent Technologies GmbH, Waldbronn, Germany), and its content quantified by NanoDrop ND-1000 (Thermo Fisher Scientific, Wilmington, USA). For RT-qPCR, double strand cDNA was synthesized from $1 \mu \mathrm{g}$ of total RNA using the iScript ${ }^{\mathrm{TM}} \mathrm{cDNA}$ Synthesis kit (Quanta Biosciences, Gaithersburg, USA) according to the manufacturer instructions. RT-qPCR was performed in a CFX96 instrument (BioRad, Hercules, CA, USA) using a 
SYBR Green master mix. Relative gene expression levels were calculated with the 2- $\Delta \Delta \mathrm{Ct}$ method, using HPRT1 and $18 \mathrm{~S}$ ribosomal RNA as reference genes. Post-run analyses were performed using Bio-Rad CFX Manager version 2.0 and the threshold cycles (Cts) were calculated from a baseline subtracted curve fit. See Supplementary Table 1 for primer pair sequences.

\subsection{Microscopy and Quantification}

Live phase contrast images from PC12 cells under the different conditions were acquired using a Nikon Eclipse Ti Inverted Microscope (Nikon; Düsseldorf, Germany) equipped with a Perfect Focus System (PFS) and a Digital cooled Sight Camera (DS-QiMc; Nikon, Germany) as described in (Weber et al., 2013). Briefly, PC12 cells were cultured in collagen coated 6-well plates (500,000 cells/well) and treated as described in "Cell culture and stimulation" and 150 images per well, every second day were recorded with the same spatial pattern. Cell differentiation is calculated by the ratio of the two described imaging features (Weber et al., 2013) convex hull (CH) to cell area (CA) for 150 images per well over 6 days (Weber et al., unpublished data).

\subsection{Western Blot}

For each timepoint and condition $3 \times 10^{6} \mathrm{PC} 12$ cells (for inhibition experiments) or $5 \times 10^{6} \mathrm{PC} 12$ cells (for EGF vs. NGF comparison) were seeded in $10 \mathrm{~cm}$ collagen coated Cell BIND dishes (Corning; Germany). Cells were collected after 5, 10, $30 \mathrm{~min}, 1,2,4,6,8,12,24$, and $48 \mathrm{~h}$ in $200 \mu \mathrm{l}$ RIPA buffer (containing $0.5 \%$ SDS), supplemented with proteinase inhibitor (complete mini EDTA free tablets, Roche, Basel, Switzerland) and Benzonase (Merck), and lysed for $20 \mathrm{~min}$ under agitation. A total of $30 \mu \mathrm{g}$ protein was loaded per lane and run in 10\% SDS- polyacrylamide gels, transferred to polyvinylidene difluoride membranes. Membranes were cut horizontally into fragments according to the expected sizes of the protein of interest and immunoblotted with antibodies against total p44/42 (ERK1/2, 1:2000, \#9102S, Cell Signaling Technology [CST]), phospho p44/42 (pERK1/2, 1:2000, \#9101S, CST), total JNK (JNK1/2, 1:1000, \#9258S, CST), phospho JNK (Thr183/Tyr185, 1:1000, \#4668S, CST), total AKT (1:1000, \#4691S, CST), phospho AKT (1:1000, Ser473, \#9271S, CST) or GAPDH (1:2000,\# MAB374, Millipore) overnight at $4^{\circ} \mathrm{C}$. Proteins were visualized with chemiluminescence on SuperSignal West Pico Chemiluminiscent Substrate imager (Thermo-Fischer, Massachusetts, USA) after $1 \mathrm{~h}$ of incubation with appropriate horseradish peroxidase-linked secondary antibody (SigmaAldrich). Immunoblots were quantified using ImageJ (image analyzer camera LAS4000, Fujifilm, Tokyo, Japan). Blots were normalized to total GAPDH and an internal standard (IS) was used for normalization between membranes.

\subsection{Microarray Analysis and Data Pre-processing}

Time-resolved gene expression data of stimulated PC12 were recorded at $t=[1,2,3,4,5,6,8,12,24] \mathrm{h}$ and $t=[1,2,3,4,6,8,12,24] \mathrm{h}$ for NGF and EGF stimulation, respectively. Control timepoints were measured at $0,2,4,6,8,12,24 \mathrm{~h}$. Total RNA was isolated, labeled and hybridized to an Illumina RatRef-12 BeadChip (Illumina, San Diego, CA, USA) according to the manufacturers protocol. Raw microarray data were processed and quantile normalized using the Bioconductor $\mathrm{R}$ package beadarray (Ritchie et al., 2011). Illumina Probes were mapped to reannotated Entrez IDs using the Illumina Ratvl annotation data (v. 1.26) from Bioconductor. If several probes mapped to the same Entrez ID, the one having the largest interquartile range was retained. This resulted in 15,348 annotated genes, whose expression was further batch corrected according to their chip identity (Johnson et al., 2007). Finally, gene expression time series were smoothed by a 5 th order polynomial to take advantage of the high sampling rate and replicates at 0,12 , and $24 \mathrm{~h}$. Microarray data have been deposited at Gene Expression Omnibus (GEO) under the accession number GSE74327.

\subsection{Multi-Dimensional Scaling}

To determine significantly regulated genes over time we performed a multi-dimensional scaling (MDS) using the HiTMDS algorithm (Strickert et al., 2005). The algorithm projects the $15348 \times 15348$ distance matrix $D$ of the pairwise Euclidean distances between all genes onto a two dimensional space, while preserving distances in $D$ as best as possible. Genes varying strongly and uniquely over time will appear as outliers in the MDS point distribution. The uniqueness of a gene expression profile was quantified by fitting a two-dimensional skewed Gaussian distribution (Azzalini, 2015) to the MDS point density function.

\subsection{Clustering Gene Expression Patterns}

To cluster the gene timeseries, we applied the Cluster Affinity Search Technique (CAST), which considers the genes and their similarity over times as nodes and weighted edges of graph, respectively (Ben-Dor et al., 1999). All clusters are considered as unrelated entities and there is no pre-defined number of clusters. Instead a threshold parameter, here $t=0.8$, determines the affinity between genes and this the final number of gene clusters. Inverse or anti-correlative behavior of genes after NGF or EGF stimulation was determined by fitting a linear model to the smoothed gene expression. Genes having a significant slope with opposite sign and an $r^{2}>0.7$ were taken as anti-correlated.

\subsection{Enrichment Analysis of Transcription Factor Target Gene Sets}

Upstream analysis for putative transcription factors regulating the EGF and NGF transcriptome responses over time were assessed by a Gene Set Enrichment analysis (Luo et al., 2009) using paired control to treatment samples for each timepoint with an overall cutoff $q$-value $<0.01$. As gene sets we used the transcription factor target lists from the Molecular Signatures Database (MSigDB, version 5.0) (Subramanian et al., 2005), for which we mapped the human genes to the rat orthologs using BiomaRt (Huang et al., 2014). 


\subsection{Boolean Model}

We used a Boolean model framework for dynamic analysis of PC12 cell differentiation. Based on our microarray data and literature knowledge we constructed a highly connected prior knowledge network (PKN) consisting of 63 nodes and 109 edges (cf. Supplementary Table 2). The R/Bioconductor package CellNetOptimizer (CNO) (Saez-Rodriguez et al., 2009) was used to optimize the PKN by reducing redundant nodes, unobservable states and edges. For this we rescaled the qRT-PCR fold change values between 0 and 1 and then transformed with a Hill function $f(x)=\frac{x^{n}}{x^{n}+k^{n}}$ as suggested in Saez-Rodriguez et al. (2009), where $n=2$ and $k=0.5$ denote the Hill coefficient and the threshold, above which a node is considered "on," respectively. Changing the Hill coefficient between $1 \leq n \leq 6$ did not change the results qualitatively. Model topology optimization was performed via the CellNORdt, which allows fitting with time course data. (See Supplementary Table 3 for stimulus, inhibition and time course data). We set the maximal CPU run time for the underlying genetic algorithm (GA) to $100 \mathrm{~s}$ and the relative tolerance to 0.01 , using default parameters from the $\mathrm{CNO}$ otherwise. A representative evolution of the average and best residual error in a GA run is depicted in Supplementary Image 1A. The solutions quickly converge to a quasi steady state within the time window of simulation of $100 \mathrm{~s}$. The following edges were fixed to prior to optimization based on literature knowledge: NGF $\rightarrow$ PI3K, NGF $\rightarrow$ RAS, NGF $\rightarrow$ PLC, AP1 $\rightarrow$ NPY, MEK/ERK \& JNK $\rightarrow$ Jund, MEK/ERK \& JNK $\rightarrow$ Junb, Fosl1 \& Jund $\rightarrow$ AP1, Mmp10 $\rightarrow$ RAS, RAS $\rightarrow$ MEK, PLC $\rightarrow$ MEK. Model optimization was performed 100 times and edges were retained, if they appeared in $70 \%$ of the runs. This cutoff was chosen to generate a sparse network with robust edges. Performing more runs did not change the results qualitatively (cf. Supplementary Image 1B). Model simulations were performed using the R/Bioconductor package BoolNet (Müssel et al., 2010). The reference publications from which the interactions have been inferred as well as their Boolean transition functions are listed in Supplementary Table 4.

\section{RESULTS}

\subsection{Gene Response of PC12 Cells Diverges for NGF and EGF on Long Time Scales}

To elucidate the dynamic gene response of NGF and EGF, we measured the transcriptome dynamics using Illumina RatRef12 Expression BeadChips. PC12 cells were either stimulated with NGF or EGF, and collected at the following timepoints: $1,2,3,4,5,6,8,12$, and $24 \mathrm{~h}$. The unstimulated control samples (ctrl) were collected in parallel. Gene expression time series were smoothed by a 5 th order polynomial to take advantage of the high sampling rate. Finally, we mapped array probes to their respective Entrez IDs, resulting in 15,348 annotated genes.

A bi plot of the principal component analysis (PCA) for the 1000 most varying genes depicted a clear separation of the control, NGF and EGF samples. The PCA scores, representing the NGF and EGF treated samples, showed a qualitatively similar behavior up to $4 \mathrm{~h}$ after stimulation, yet differed markedly beyond that time (Figure 1A, left). The absolute length and direction of the PCA loadings (Figure 1A, right) indicate the contribution of individual genes to the position of the scores. Correspondingly, several immediate early genes, such as Junb (Jun B ProtoOncogene), Fos (FBJ Murine Osteosarcoma Viral Oncogene Homolog), Ier2 (Immediate Early Response 2), and Egr1 (Early Growth Response 1) contributed to the early gene response under both EGF and NGF stimulation, while members of the uPAR/Integrin signaling complex, such as Mmp13/10/3 (Matrix Metallopeptidase 13/10/3), Plat (Plasminogen Activator, Tissue) and Serpine1 (Serpin Peptidase Inhibitor, Clade E, Member 1) determined, among others, the separation of the NGF from the EGF trajectory. Loadings that point toward the control and late EGF response samples, like Cdca7 (Cell Division Cycle Associated 7) and G0s2 (G0/G1 Switch 2), are clearly related to cell cycle progression and additionally highlight the difference in proliferation vs. differentiation. In conclusion, the NGF gene response, and thus PC12 cell differentiation, must be determined by late transcriptional feedback events, that trigger and sustain MAPK/ERK signaling.

Next, we sought to functionally analyze the transcriptional differences in early and late gene regulation after EGF and NGF stimulation. For this we selected genes that are (i) strongly regulated $\left(\log _{2}\right.$ fold change of $<-1.7$ or $>1.7$ in two consecutive timepoints) and (ii) have a unique temporal expression profile according to a multi-dimensional scaling (MDS) analysis ( $p$-value $<0.01$ ) (cf. Supplementary Image 2). We found 152 and 402 genes, meeting both criteria, in the EGF and NGF data, respectively, among which 126 genes are shared by both conditions. Figure 1B depicts a clustering of these differentially i.e., top-regulated genes. A cluster affinity search technique (Ben-Dor et al., 1999) identified five EGF (E1-E3b) and seven NGF (N1-N4B) gene response clusters (cf. Supplementary Table 5 and Supplementary Image 3). Interestingly, the EGF stimulus induced a short pulse-like response with rapid return to original gene expression levels, while the NGF stimulus induced a combination of short-impulse like (N1 - N2b) and long sustained gene expression patterns with several clusters (N3a$\mathrm{N} 4 \mathrm{~b}$ ) sustaining their expression over time (cf. circled insets in Figure 1B).

Figure 1C depicts a network representation of the enrichment analysis using a hypergeometric test on Gene Ontologies (GO). Enriched upregulated biological functions were identified in gene lists E1, E2a, N1, N2a, N3a, N4a and in both groups of inversely regulated genes (cf. Supplementary Table 6). Nodes correspond to GO terms, with numbers indicating the joint enrichment scores. Nodes sharing at least 20 percent of their genes are connected by solid or dotted edges, if the connected nodes lie within a stimulus or across NGF and EGF treatment. Early transcription factor activity is common to both, NGF and EGF signaling, (clusters E1 and N1) as well as MAPK signaling genes (clusters E2a and $\mathrm{N} 3 \mathrm{a}$ ). The latter, however, is more prominent and enriched at later points in time after NGF stimulation (N3a) compared to the EGF induced response (E2a). Here, a less and earlier enrichment of MAPK signaling genes was seen. Moreover, a second network of transcription factor activity could be identified after NGF stimulation (cluster $\mathrm{N} 2 \mathrm{a}$ ) that does not have any equivalent after EGF stimulation. It seems, that the initial response (first hour) is 
A

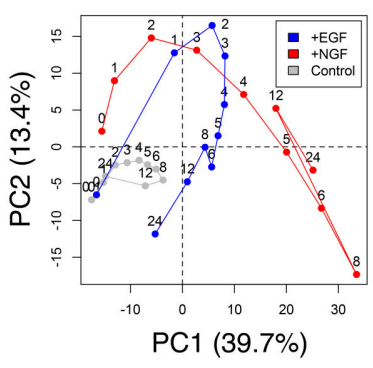

PC1 (39.7\%)

B

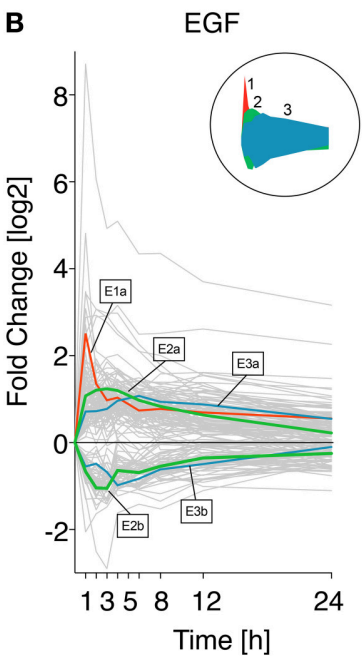

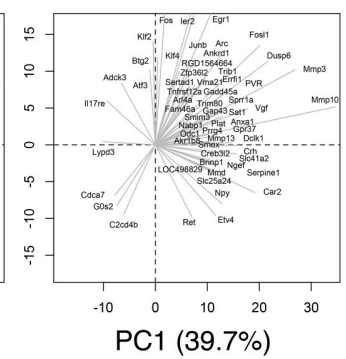

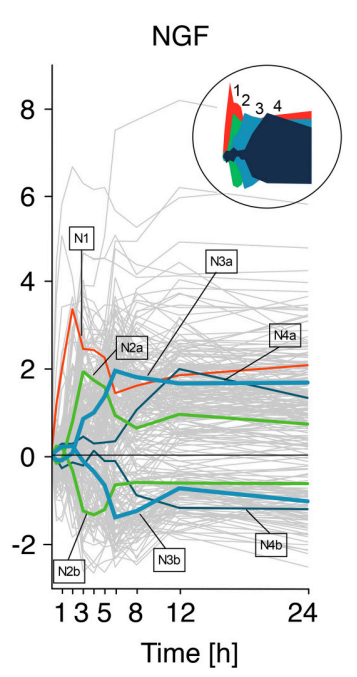

C

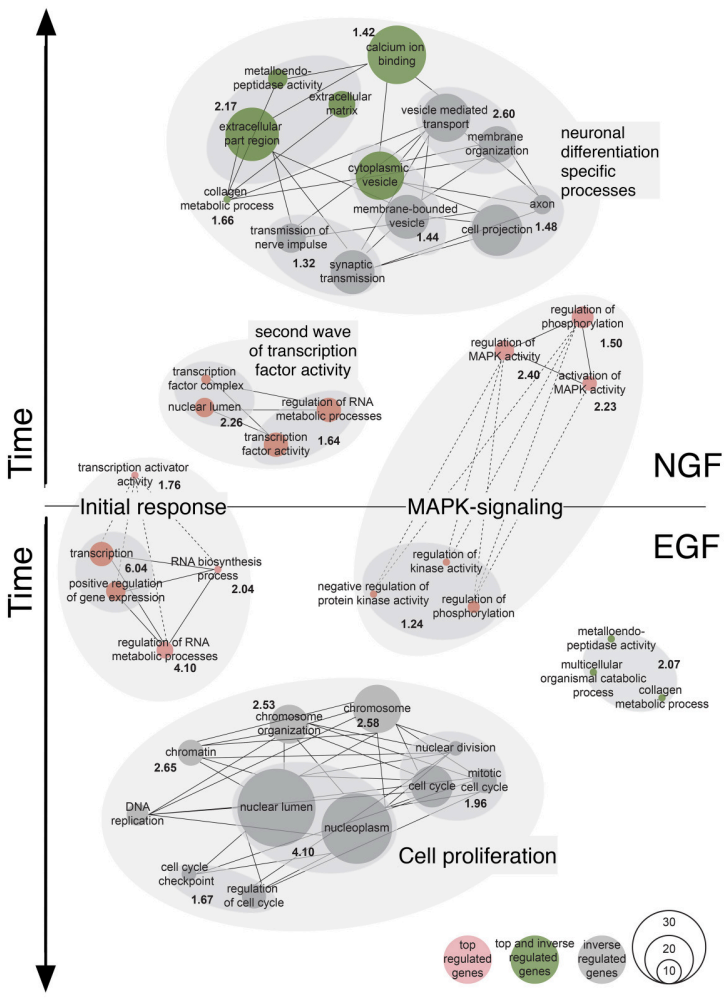

FIGURE 1 | Gene response dynamics after NGF or EGF stimulation. (A) Principal component analysis (PCA) of the PC12 cell transcriptomes after NGF (red), EGF (blue) and control treatment (gray). The PCA scores (left panel) and loadings (right panel) correspond to the samples and genes, respectively. Samples in the left panel have been connected to guide the eye. Clearly, EGF and NGF samples remain close in the first $3 \mathrm{~h}$ and separate at later timepoints, indicating a different cellular phenotype. Right panel: 50 largest loading vectors indicating the impact and time of action of individual genes. Immediate early genes, like Fos or Ier2 point toward early timepoints, while loadings pointing toward the right, like $V g f$ or $N p y$, correspond to late timepoints and are most likely involved in differentiation. (B) Expression clusters of top regulated genes. The left and right panels depict the response of individual genes to EGF and NGF stimulation, respectively (gray lines). Cluster centroids are marked by lines with the cluster size encoded by line thickness. The circular inserts depict the cluster centroid envelopes for EGF and NGF, respectively. (C) Network representation of functional enrichment of NGF and EGF response genes. The network is comprised of GO-term clusters having a significant enrichment $\left(-\log _{10}(p\right.$-value $\left.)>1.3\right)$ as shown in bold black numbers. Red, gray and green nodes contain in this order top-regulated genes, inversely-regulated genes between EGF and NGF or both. The vertical node location corresponds to the peak regulation of their genes, while node size is proportional to the number of genes in a functional category. Edges indicate a gene overlap of $>20 \%$ between nodes, being drawn as dashed lines, if they are shared between EGF and NGF.

controlled by a shared set of top-regulated genes (cf. Figure 1C, dashed lines). The cell-fate specific processes, however, seem to be orchestrated by different set of genes (cf. Figure 1C, separate networks). Many of the genes executing proliferation or differentiation specific processes fall into the category of inversely regulated genes and are not amongst the set of topregulated genes identified earlier (cf. Figure 1C, green and gray nodes, cf. Material and Methods, cf. Supplementary Table 7). The genes involved in the procession of extracellular matrix and cytoplasmic vesicles, however, constitute an exception: these genes are both top and inverse-regulated (cf. Figure 1C, green nodes).

In summary, functional analysis of the gene clusters revealed an initiation of the differentiation and proliferation process by a shared set of differentially regulated genes. Specific functions, such as transmission of nerve impulse or DNA replication, however, seemed to be executed by two distinct gene groups that are when comparing the EGF to the NGF stimulus inversely regulated over time. Additionally, a second network of genes involved in transcription factor activity was identified in the NGF data set, which lacked a corresponding network in the EGF data set.

\subsection{Simulation of a Boolean Network}

Based on the above gene response analyses we sought to identify the mechanisms that sustain MAPK signaling activity after NGF stimulation. Our transcriptome timeseries analysis revealed that the decision process between proliferation and differentiation was spread out over several hours during which transcriptional feedback through an additional set of transcription factors was present after NGF stimulation, only (cf. Figure 1C). To further elucidate the transcription factors upstream of the gene 
response after EGF or NGF stimulation we performed a gene set enrichment analysis (GSEA) (Luo et al., 2009) on the paired NGF to control and EGF to control transcriptome timeseries. As gene sets we used the motif gene sets from the Molecular Signatures Database (MSigDB v5.0) (Subramanian et al., 2005) and mapped the human genes onto the rat orthologs using BiomaRt (Huang et al., 2014).

Figure 2A compares the temporal significance of transcription factors for EGF and NGF stimulation. EGF elicited an early, yet transient significance of all transcription factors, while the time-resolved transcription factor significances for NGF showed early, transient and late activity. Figure 2B depicts the differences in TF significance between NGF and EGF. The most down-regulated TFs relative to EGF are E2F1, EBF1, SOX9 and SP1, all of which are linked to cell proliferation (Bastide et al., 2007; Hallstrom et al., 2008; Györy et al., 2012; Zhang et al., 2014).

Mullenbrock et al. (2011) showed late NGF-induced genes up to $4 \mathrm{~h}$ were preferentially regulated by AP1 and CREB (cAMP response element-binding protein). While AP1 was among the most persistently up-regulated transcription factors, we found a transient significance for CREB1, only, peaking at 3 and $6 \mathrm{~h}$, under EGF or NGF stimulation, respectively, which indicated the importance of further TFs beyond that time window. In fact, we found the highest positive differences in the transcription factors $\mathrm{BACH} 2, \mathrm{AP} 1$, as well as ELF2 and ETV4. The latter two belong to the ETS transcription factor family. In particular ETV4, a member of the PEA3 subfamily of ETS, has been shown to promote neurite outgrowth (Fontanet et al., 2013; Kandemir et al., 2014). BACH2, member of the BTB-basic region leucine zipper transcription factor family, is known to downregulate proliferation and is involved in neuronal differentiation of neoblastoma cells via p21 expression (Shim et al., 2006) and it interacts with the transcription factor MAFF (V-Maf Avian Musculoaponeurotic Fibrosarcoma Oncogene Homolog F) (Kannan et al., 2012) that is necessary for differentiation.

To analyze the early cellular response upon treatment, we additionally compared the phosphorylation levels of pERK, pAKT and pJNK under NGF and EGF stimulation over time (Figure 2C). As expected, pERK increased after NGF and EGF stimulation, showing a persistent up-regulation for $8 \mathrm{~h}$ or pulse-like response, respectively. pJNK was continuously upregulated under NGF relative to EGF stimulation, whereas pAKT responded similar to both stimuli, yet showed a consistently higher phosphorylation under EGF beyond $2 \mathrm{~h}$. Taken together, this corroborates the roles of both pERK and pJNK as well as pAKT in PC12 cell differentiation and proliferation, respectively (Waetzig and Herdegen, 2003; Chen et al., 2012).

Based on the combined transcriptome, upstream transcription factor and protein analyses we next developed a comprehensive prior knowledge interaction network (PKN) for NGF induced PC12 cell differentiation. The PKN comprises key players of known pathways involved in PC12 cell differentiation, such as ERK/PLC/PI3K/JNK/P38/uPAR/NPY and integrin signaling, as well as "linker nodes" to obtain a minimal, yet fully connected network, consisting of 63 nodes and 109 reactions (cf. Supplementary Table 4 for reference publications). The network is depicted in Supplementary Image 4 with differentially regulated genes obtained from our timeseries marked in red and points of inhibition indicated by orange. A Cytoscape readable network format is provided in Supplementary Table 2. Albeit the included PKN pathways are much more complex, our focus was on simulating a biologically plausible signaling flow, including protein signaling, gene response and autocrine signaling as follows: stimulated TrkA receptor activates the downstream pathways PLC/PKC, MAPK/ERK, PI3K/AKT, and JNK/P38. Phosphorylated ERK, PI3K and P38/JNK together activate different transcription factors such as Fosl1, Fos, Junb, Btg2, Klf2/5/6/10, Cited2, Maff, and Egr1, which are important for PC12 cell differentiation according to our analysis and literature (Cao et al., 1990; Ito et al., 1990; Levkovitz and Baraban, 2002; Gil et al., 2004; Eriksson et al., 2007).

Junb and Fos initiate the AP1 system, which in turn induces uPA/uPAR signaling, triggering the formation of plasmin (Avraham and Yarden, 2011). The latter is a major factor for the induction of $M m p 3 / M m p 10$, linking degradation of the extracellular matrix (ECM) with integrin signaling. The integrins transmit extracellular signaling back via the focal adhesion kinase (FAK) (Singh et al., 2012). FAK activates again the SHC protein, which closes the autocrine signaling. Previous studies reported that uPAR expression is necessary for NGF-induced PC12 cell differentiation (Farias-Eisner et al., 2000; Mullenbrock et al., 2011). A second autocrine signaling loop in the initial PKN putatively acts via the AP1 system, which in turn activates the Neuropeptide Y (NPY/NPYY1 pathway). NPY is a sympathetic co-transmitter that acts via $\mathrm{G}$ protein-coupled receptors through interactions with its NPYY1 receptors (Selbie and Hill, 1998; Pons et al., 2008). NPYY1 receptor further activates $\mathrm{Ca}^{2+}$ dependent PKC /PLCgamma and subsequently convergences to ERK signaling.

To optimize the highly connected PKN we used CellNetOptimizer (CNO) (Saez-Rodriguez et al., 2009). The CNO first compresses the network, i.e., it deletes unobservable nodes and then optimizes the network topology using a genetic algorithm. We trained the PKN using gene expression of selected differentially regulated genes under NGF stimulus and inhibition of either MEK, JNK, or PI3K (Figure 3A, MEKi, JNKi and PI3Ki). The overall gene response showed a gradual decline in fold change from NGF via MEK to JNK inhibition, while inhibition of PI3K only moderately impacted the gene expression (Figure 3A). The most affected genes under MEK and JNK inhibition were members of the UPAR signaling pathway, Mmp10, Mmp3, and Plaur as well as the transcription factors Fosl1 and Egr1, Plaur, Dusp6 (Dual Specificity Phosphatase 6) and lastly Npy.

Topology optimization using the above perturbations led to a greatly reduced network. Optimization lumped linear pathways into one node, such as the autocrine feedback via uPA/PLAT to Itgal and FAK or MEK to ERK transition. The reduced network revealed both MAPK/ERK and JNK as the central network hubs, distributing the upstream signals to downstream genes. It includes two positive feedback via AP1 and uPAR signaling back to FAK and MAPK as well as AP1 to Npy and PKC/PLC back to MAPK. To comply with prior knowledge, we re-expanded linear 
A

A

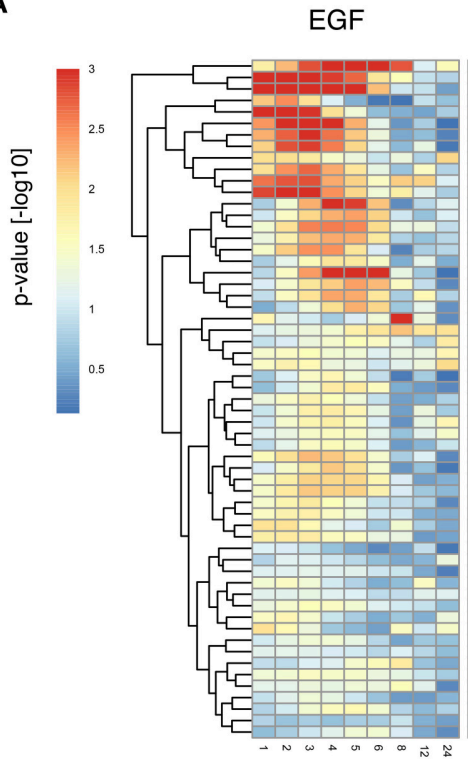

Time [h]

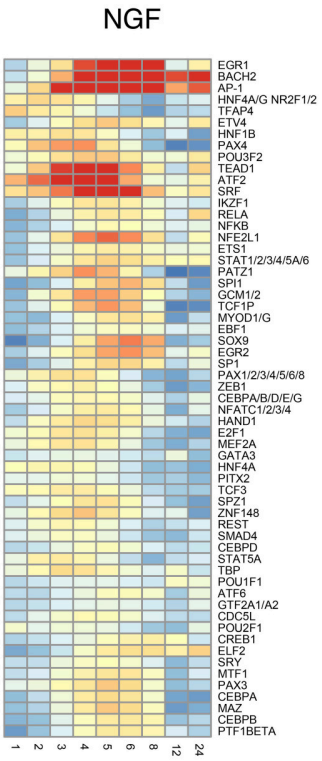

Time [h]
B Difference NGF-EGF

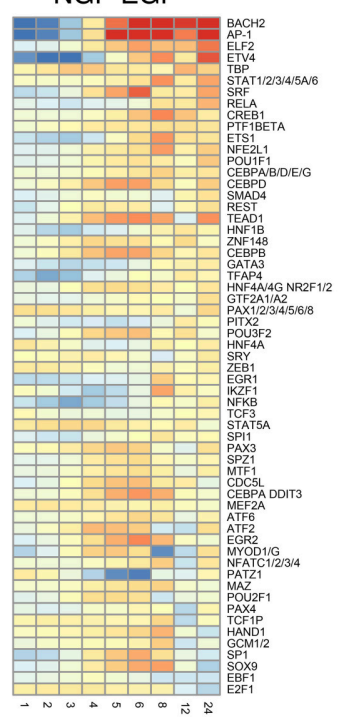

Time [h]

C
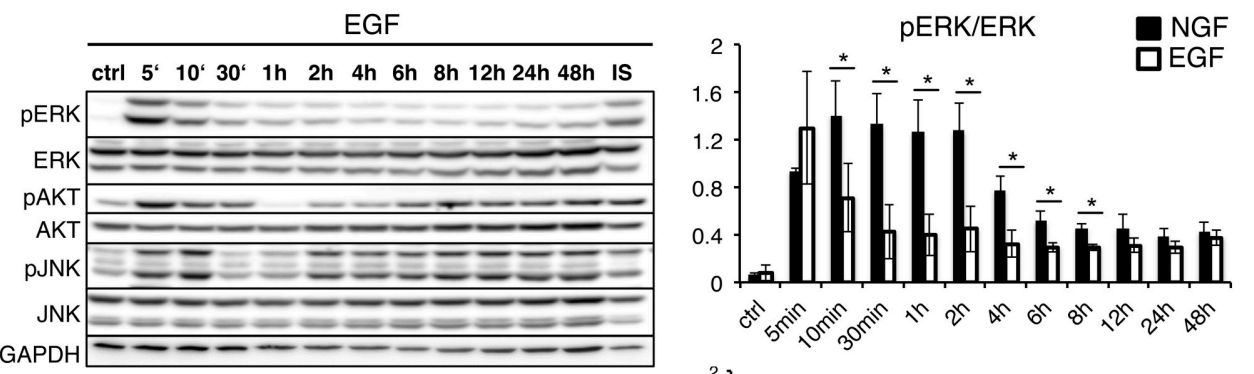

NGF

ctrl $5^{\prime} 10^{\prime} 30^{\prime}$ ih $2 \mathrm{~h} \quad 4 \mathrm{~h} 6 \mathrm{~h} \quad 8 \mathrm{~h} 12 \mathrm{~h} 24 \mathrm{~h} 48 \mathrm{~h}$ IS
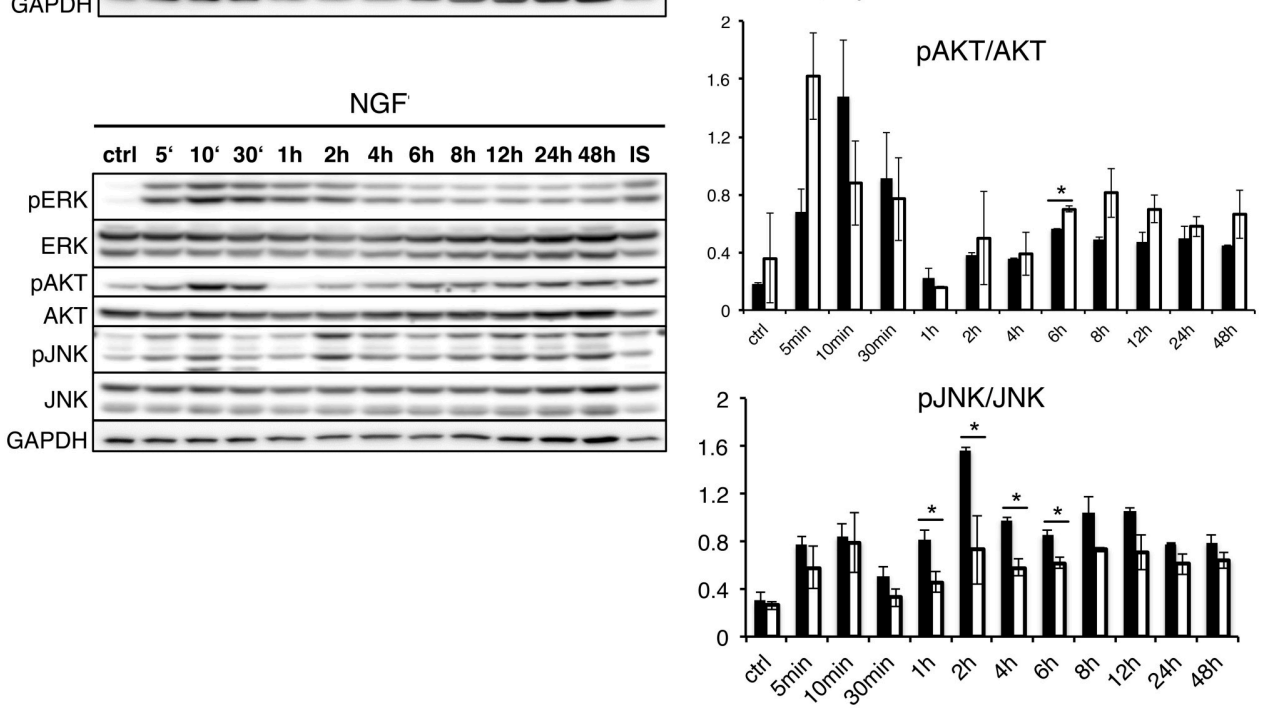

FIGURE 2 | Upstream analysis of gene expression timeseries. (A) Upstream Gene Set Enrichment Analysis for transcription factors. The heatmaps depict the significance of transcription factors putatively controlling the gene response after EGF (left) or NGF stimulation (right). All TFs are significantly regulated (FDR corrected $p$-value < 0.01) after NGF treatment. TFs have been clustered by their Euclidean distance across all conditions using a complete linkage method. (B) Difference in TF $p$-value significance (NGF-EGF). Rows were ordered from the most positive to the most negative difference at $t=12$ and $24 \mathrm{~h}$. (C) Time-resolved quantification of pERK, pAKT and pJNK after EGF and NGF treatment. Original western blots from PC12 cells treated with 75 ng/ml EGF and 50 ng/ml NGF over time. GAPDH is shown as loading control, IS: Internal Standard. Statistical analysis of the pERK/ERK, pAKT/AKT and pJNK/JNK levels are shown on the right panel. An increased and significant higher pERK/ERK level is shown in NGF stimulated (shown as black bars) cells compared to EGF (shown as white bars). A similar trend is visible for pJNK/JNK. A * denotes a $p$-value $<0.05$, data points obtained in duplicates and triplicates. 


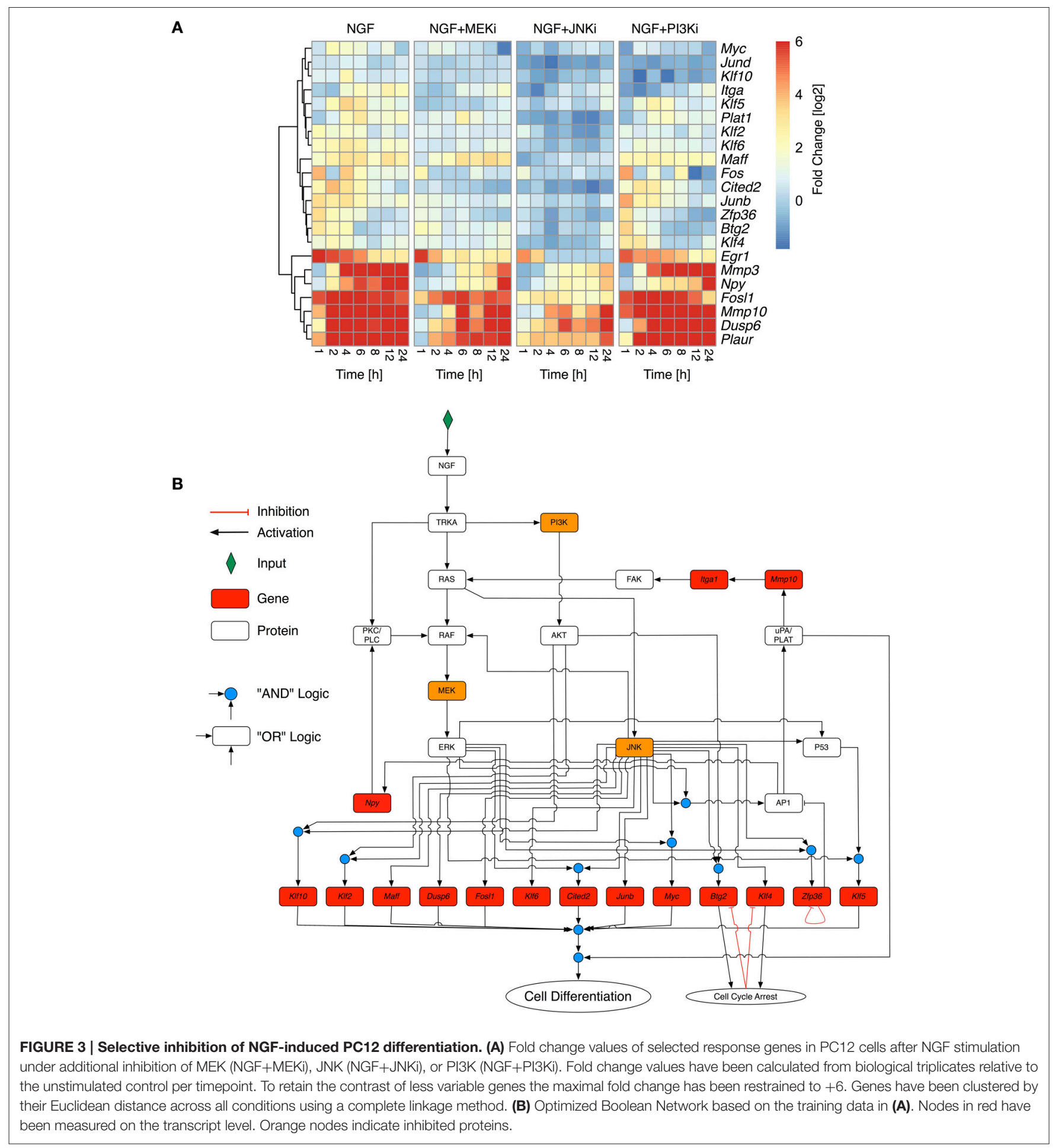

pathways and added known down-stream target genes, such that the final network, shown in Figure 3B, comprised 32 nodes and 52 edges. We assumed that PC12 differentiation occurs, if the majority of these genes is activated together with uPAR signaling. Due to the inherent difficulty of Boolean networks to incorporate negative feedback loops, we revised the network topology of the reduced network to include transient gene activity of several moderately responding genes. Klf4 and Btg2 have been previously been indicated as immediate early genes in PC12 cell differentiation (Dijkmans et al., 2009) and are involved in growth arrest (Tirone, 2001; Yoon et al., 2003), which is a necessary prerequisite for differentiation and degradation of 
mRNA, respectively. While the explicit mechanism of how Klf4 and Btg 2 are regulated remains unclear, we assumed an autoinhibition once they mediated their growth arrest effect. Zfp36 belongs to the TTP (Tristetraprolin) family of proteins and has been shown to degrade AU-rich mRNAs, particularly of early response genes (Amit et al., 2007). It negatively regulates its own expression (Tiedje et al., 2012) and therefore in the model effectively delays the activity of AP1 before switching itself off. Of note, another member of the TTP protein family, Zfp3612 (zinc finger protein $36, \mathrm{C} 3 \mathrm{H}$ type-like 2) is constitutively expressed at long times after NGF stimulation (data not shown) and might act as another long-term negative feedback regulator and causing downregulation of Egr1, Fos, and Junb. Indeed, our experimental data revealed a reduction on gene expression of Egr1, Fos and Junb over time (Figure 3A).

We simulated the optimized and re-expanded Boolean network (cf. Supplementary Table 8) using the BoolNet $\mathrm{R} /$ Bioconductor package (Müssel et al., 2010), performing two types of simulations. First, we tested the robustness and alternative attractors by setting NGF to "on" and randomly initializing all other network nodes. The nodes were then synchronously updated until a steady state was reached. Within $n=10^{7}$ different simulations, the same final network state with "cell differentiation" set to "on" was always reached. Although this was not an exhaustive search given the number of possible initial network states, it still demonstrated the robustness of the network output. Next, to show the information flow from the NGF receptor to the downstream nodes under different inhibitory conditions, we initialized all nodes except NGF to "off" and performed synchronous updates until a steady state was reached (Figure 4A). Without inhibition, NGF sequentially switches on MAPK, AKT and JNK pathways as well as uPAR signaling. Klf4, Btg2, and Zfp 36 become transiently active, with the latter delaying AP1 activity. Blocking MEK (NGF+MEKi) inhibited ERK and thus several downstream targets, including the uPAR feedback. As the latter is assumed indispensable for PC12 cell differentiation, (Farias-Eisner et al., 2000, 2001), the model predicted inhibition of PC12 cell differentiation. The same phenotype is found, when blocking JNK (NGF+JNKi). In comparison to NGF+MEKi it even abrogated the activity of downstream targets altogether. Inhibition of PI3K (NGF+PI3Ki) solely affected PI3K and its downstream target protein AKT and target genes Maff and Klf10, yet cell differentiation persisted.

Taken together, we developed a core network from the downstream interactome of PC12 cell pathways involved in differentiation. The model captured the dynamic pathway activation after NGF stimulation and various inhibitions. It assigned central and synergistic roles for ERK and JNK in PC12 differentiation with JNK having the largest impact on the network activity.

\subsection{Model Analysis and Experimental Confirmation}

Network simulations were confirmed by live phase-contrast imaging (Figure 4B) and western blot analyses (Figure 5). We measured the convex hull $(\mathrm{CH})$ to cell area $(\mathrm{CA})$ ratio of $\mathrm{PC} 12$ cells on days 2, 4, and 6. A large convex hull due to extended neurite (marked as red arrow heads in Figure 4B) and small overall cell area is indicative of differentiation (Figure 4B, right panel). Clearly, the continuous $\mathrm{CH} / \mathrm{CA}$ ratio at day 2 was largest for NGF stimulation and NGF stimulation with additional PI3K inhibition, which corresponded well with the cell differentiation set to "on" in the network simulations under these condition. One can speculate whether inhibition of the pro-proliferative PI3K pathway amplifies cell differentiation, possibly relieving a negative feedback. Indeed, a Western blot of the pERK/ERK ratio depicted a trend to higher ERK phosphorylation relative to NGF stimulation under PI3K inhibition (Figure 5) and phasecontrast images of PC12 cells show more and longer neurites in comparison to cells treated only with NGF or in combination to MEKi and JNKi (Figure 4B, NGF+PI3Ki). Interestingly, image analysis suggested not a stop, but rather a delay of cell differentiation under MEK inhibition. In detail, PC12 cells show no neurites under MEKi after 2 days of combined NGF treatment compared to NGF alone or NGF-PI3Ki. After 4 and 6 days of NGF+MEKi treatment, less cells have neurites in comparison to cells that were only treated with NGF (Figure 4B, NGF+MEKi). In line with literature, pERK levels were reduced, yet pJNK levels were likewise increased, indicating a redirection of protein activity under MEK inhibition (Figure 5, right panel). Likewise, the gene expression showed a reduced, but not completely abolished fold change for Mmp10 (Figure 3A) and also an upregulation of Dusp6. Although the discrete Boolean model could not simulate gradual responses, MEK inhibition still resulted in the activation of several downstream target genes necessary for PC12 cell differentiation, while none of these were active under JNK inhibition. In summary, modeling and simulation suggested that PC12 differentiation involved the activity of both JNK/JUN, MAPK/ERK and PI3K/AKT signaling pathways. The establishment of a positive, autocrine feedback loop was indispensable to active late and persistent gene expression.

\section{DISCUSSION}

PC12 cells are a well established model to study the cellular decisions toward proliferation or differentiation. Nevertheless, there is still a lack of understanding on how protein signaling and gene regulation interact on different time scales to decide on a long-term, sustained phenotype. Given the fact that PC12 cell cycle and differentiation last up to 4 and 6 days, respectively (Greene and Tischler, 1976; Luo et al., 1999; Adamski et al., 2007), late events occurring beyond the first hours are most likely to be important for sustaining the cellular decision. However, few studies that have compared the long-term effect of EGF and NGF in PC12 cells. They focused either on NGF alone (Dijkmans et al., 2008, 2009), on individual (Angelastro et al., 2000; Marek et al., 2004; Lee et al., 2005; Chung et al., 2010), or early time-points (Mullenbrock et al., 2011).

Previous studies have identified expression of immediate early genes (IEG), such as Egr1, Junb, and Fos together with delayed early genes (DEG), like Dusp6, Mmp3/10, Fosl1, and Atf 3 as necessary for PC12 cell differentiation (Vician et al., 
A
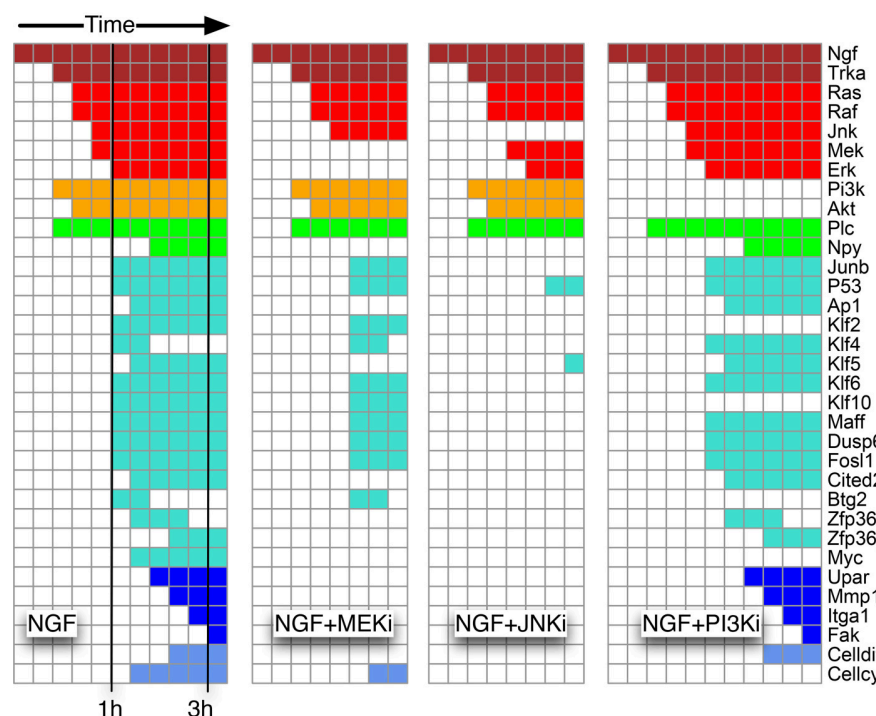

Ras
Raf
Jnk
Mek
Erk

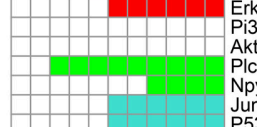

Pi3k
Akt
Plc

Npy
Junb
P53

Junb
P53
Ap1

Ap1
Klf2
Klf4
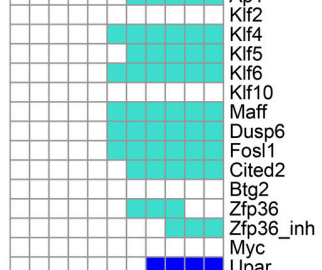

36_inh

Mmp10

$\mathrm{NGF}+\mathrm{PI} 3 \mathrm{Ki}-\operatorname{ltga} 1$

tga1

Celldifferentiation

Cellcyclearrest
B
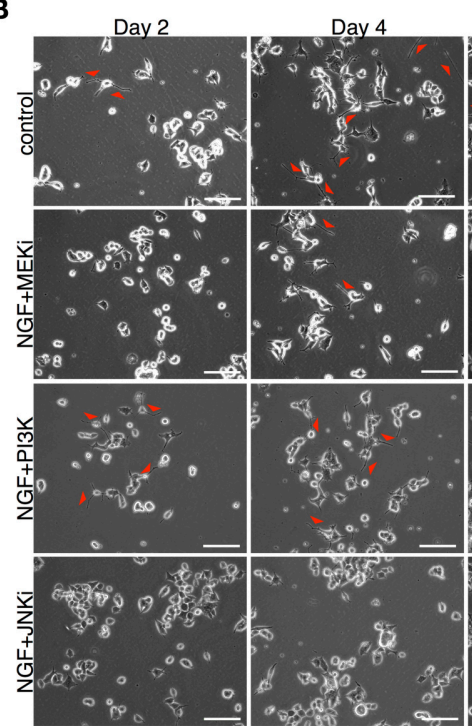
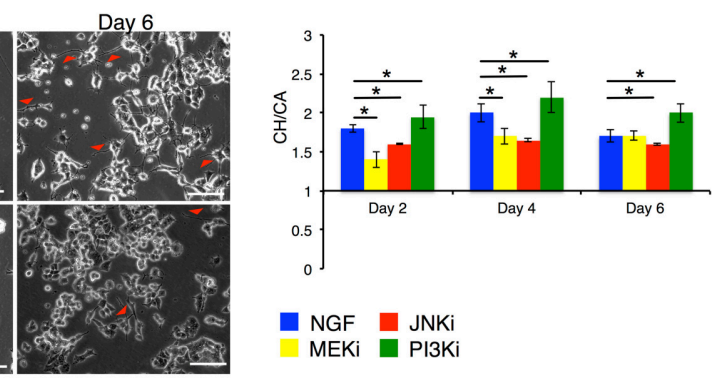

NGF JNKi

MEKi $\square$ PI3Ki

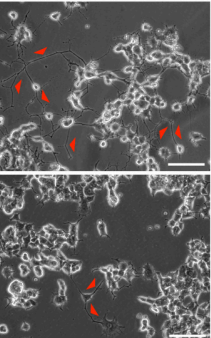

FIGURE 4 | Network simulation of time sequential pathway activation and experimental validation. (A) The heatmaps depict the path to attractor upon NGF stimulation. Columns correspond to synchronous update steps of the Boolean network. Time progresses from left to right until a steady state is reached. Initially all nodes, except NGF, are set to zero. Colored boxes correspond to activated nodes with the color denoting individual pathways/node categories. Cells are predicted to differentiate, if the node "Cell differentiation" is active, as in the case for NGF, or NGF+PI3Ki treatment. (B) Left: phase contrast images for days 2, 4, and 6 are shown for the 4 different conditions: NGF (control), NGF+MEKi, NGF+PI3Ki and NGF+JNKi. Red arrows depict sites of neurite outgrowth in differentiating PC12 cells. Bar: $100 \mu \mathrm{m}$. Right: statistical analysis of PC12 cell differentiation from phase contrast imaging for the different conditions are shown as convex hull (CH) to cell area (CA) ratio. Bars show Mean $\pm \mathrm{SEM}, n=2$, ( ${ }^{*} t$-test $p$-value $<0.05$ ).

1997; Levkovitz et al., 2001; Dijkmans et al., 2008; Mullenbrock et al., 2011). However, we found all these genes strongly regulated by both EGF and NGF stimulation (Supplementary Table 5), however, showing differences in their expression kinetics (Figure 1). Akin to differences in the pERK dynamics, these results suggest that cellular decisions toward differentiation or proliferation are driven by the differences in the gene expression kinetics.

It has been suggested before that distinct cellular stimuli activate similar sets of response genes, whose expression dynamics, rather than their composition, determine cellular decisions (Murphy and Blenis, 2006; Amit et al., 2007; Yosef and Regev, 2011). Single expression bursts are likely to stimulate proliferation, while complex, wave-like expression patterns induce differentiation (Bar-Joseph et al., 2012). Accordingly, EGF elicited a pulse-like gene response, while NGF induced a complex, wave-like gene response (Figure 1B). After EGF stimulation the expression of IEGs, Egr1, Fos, and Junb was quickly attenuated through the rapid up-regulation of their negative regulators, namely Fosl1, Atf3, Maff, Klf2, and Zfp36l2 and contributing to 


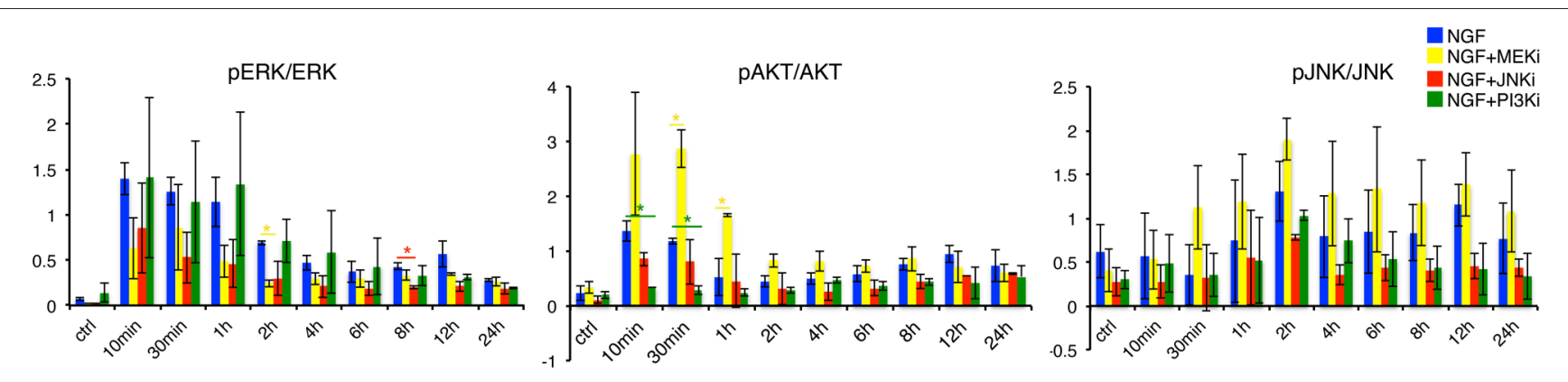

FIGURE 5 | Quantification of pERK, pAKT and pJNK levels under NGF and individual inhibitor treatments. Determination of pERK/ERK, pAKT/AKT and pJNK/JNK under NGF, NGF+MEKi, NGF+JNKi and NGF+PI3Ki treatment. Left panel: pERK/ERK levels decrease over time under NGF plus MEK and JNK inhibition. In contrast, PI3K inhibition shows a similar increase and sustained pERK/ERK levels over time compared to NGF treated PC12 cells alone. Interestingly, pAKT/AKT is increased under NGF+MEKi treatment, which is particularly significant in the early timepoints ( 30 min and $1 \mathrm{~h}$ ) compared to NGF alone or the other two inhibitors. The latter two show decreased PAKT/AKT levels over time (middle panel). A * denotes a $p$-value $<0.05$, data points obtained in duplicates and triplicates.

a pulse-like gene expression. Furthermore, Fosl1 counteracts Fos and AP1 (Hoffmann et al., 2005) and Atf3 has been shown to modulate Egr1 activity (Giraldo et al., 2012), while Maff and Klf2 negatively regulate serum response and STAT-responsive promoter elements (Amit et al., 2007). The same genes respond after NGF stimulation, however with a delayed response and might be one of the reasons for the stronger and longer gene and pERK response under NGF stimulation (Murphy et al., 2002, 2004; Murphy and Blenis, 2006; Saito et al., 2013).

A recent study by Mullenbrock et al. (2011) compared the transcriptome response of PC12 cells to EGF and NGF stimulation up to $4 \mathrm{~h}$. Using chromatin immunoprecipitation they found a preferential regulation of late genes through AP1 and CREB TFs after NGF stimulation, which is in line with our findings (Figure 2A). However, we predicted a constitutive significance for AP1 up to $24 \mathrm{~h}$, while CREB1 displayed a transient importance, being most abundant at $6 \mathrm{~h}$ after stimulation. Furthermore, we found a switch in the composition of transcriptional master regulators between 4 and $12 \mathrm{~h}$. During this time, late TFs, such as BACH2, ETS1 and ELF2 become active.

Supplementary Image 5 depicts a Volcano plot of their target genes. Beyond the early gene targets, such as Fosl1 or Junb, the late TFs additionally target related to cytoskeleton, morphogenesis and apoptosis, such as Tumor Necrosis Factor Receptor Superfamily, Member 12A (Tnfrsf12a), DoublecortinLike Kinase 1 (Dclk1), Nerve Growth Factor Inducible Vgf, Coronin, Actin Binding Protein, 1A (Coro1a, Growth Arrest And DNA-Damage-Inducible, Alpha (Gadd45a) and Npy. Of note, we found Rasa2 among the targets, which has recently been identified as a driver for differentiation through a negative feedback between PI3K and RAS (Chen et al., 2012).

A recent study by Aoki et al. (2013) investigated the downstream gene response upon light-induced intermittent and continuous ERK activation in normal rat kidney epithelial cells. Similar to the TF activity after EGF and NGF stimulation in PC12 cells, intermittent pERK activity caused up-regulation of Fos, Egfr, Ier2, and Fgf21, which were putatively controlled through serum response factor (SRF) and CREB binding sites, while sustained pERK activity caused gene regulation controlled by AP1 and BACH1. One can speculate that it is more the temporal dynamics of pERK and less the upstream ligands, such as EGF or NGF, that eventually encode the transcriptional program deciding on the cell fate.

To elucidate the various pathways and downstream target genes under NGF stimulation we constructed a Boolean model based on our transcriptome and additional literature data. A prior knowledge network revealed a highly interconnected pathway map transmitting NGF-induced signals. Training the network via inhibition of MEK, JNK or PI3K reduced the number of edges and nodes by about $80 \%$ and revealed the MAPK/JNK pathway as second signaling hub next to MAPK/ERK. Moreover, blocking the JNK pathway had a more drastic effect on cell differentiation than blocking MAPK/ERK via inhibition of MEK through UO126. Indeed, studies on the effect of MEK inhibition for PC12 cell differentiation are inconclusive. Early studies report how MEK inhibition completely averted PC12 cell differentiation (Pang et al., 1995; Klesse et al., 1999), while recent experiments suggest a decrease, rather than full inhibition of differentiation (Levkovitz et al., 2001; Chung et al., 2014). Our results were in line with the latter. Despite a significant reduction in pERK (Figure 5), our cell morphology measurements detected merely a decrease in the formation of neurites, rather than full inhibition of differentiation. The reason for this discrepancy could lie in the time scale of observation. MEK inhibition delayed differentiation and it took 6 days to eventually overcome this delay (Figure 4B). This confirmed the modeling results, which established JNK as key regulator that is closely interlinked with MAPK/ERK signaling. In concert with pERK, also pJNK becomes constitutively active upon NGF stimulation (Figure 2C). Moreover, blocking pERK through MEK even increased pJNK (and pAKT) levels, while pERK decreased after JNK inhibition, verifying a crosstalk between JNK and ERK pathways. Previous reports suggested such a crosstalk due to dual-phosphatase interaction (Fey et al., 2012), while other studies proposed that JNK phosphorylates RAF (Adler et al., 2005; Chen et al., 2012) and thereby contributing to MAPK/ERK activity. However, the mechanistic details governing 
the crosstalk remain unclear so far. In conclusion, while previous studies assigned parallel, non-redundant roles to MAPK/ERK and MAPK/JNK (Waetzig and Herdegen, 2003), our results show that JNK signaling might be even the main driver for PC12 cell differentiation.

Next to the negative feedback loops through Klf4, Zfp36, and Btg2, arresting cell cycle and attenuating mRNA abundance, we included also two positive feedback loops via uPAR and integrin signaling as well as through Neuropeptide $\mathrm{Y}$ and PKC/PLC signaling. Positive feedback loops are a common regulatory pattern in molecular biology to induce bistability switch-like behavior, particularly in cell fate decisions and differentiation (Xiong and Ferrell, 2003; Mitrophanov and Groisman, 2008; Kueh et al., 2013). In fact, multiple feedbacks deciding between PC12 cell differentiation and proliferation, have been studied on the level of MAPK signaling (Santos et al., 2007; von Kriegsheim et al., 2009). Recently, Ryu et al. (2015) used a FRET construct to quantify pERK dynamics on a single cell level after growth factor stimulation. While the cell population average still resembled the hitherto described transient and sustained pERK activity after respective EGF and NGF stimulation, the authors found a highly heterogenous response on the single cell level. Pulsed stimulation, however, not only synchronized MAPK activity between cells, but also triggered PC12 differentiation upon EGF stimulation, if the integrated pERK signal was large enough. The authors concluded that thus not only MAPK signaling, but also further pathways are responsible for the cell fate decision. Sparta et al. (2015) used a similar experimental approach to single cell response of human MCF10A-5e cells to show that EGFR activity induced a frequency modulation response, while TrkA activity caused amplitude modulation of pERK levels. The authors explained these finding by additional receptordependent signaling networks beyond the core Ras-Raf-MEKERK pathway. Extending on this idea, our data and model suggest autocrine signaling as further feedbacks that sustain the expression of differentiation inducing TFs. Indeed, uPAR and also Npy activity were strongly correlated with differentiation (Figure 3A) and neither Npy nor uPAR signaling were activated upon EGF stimulation (data not shown). In line with this finding previous studies reported that UPAR expression is necessary for NGF-induced PC12 cell differentiation (Farias-Eisner et al., 2000; Mullenbrock et al., 2011). SERPINE1 regulating the plasminogen activator-plasmin proteolysis was shown to promote neurite outgrowth and phosphorylation of the TrkA receptor and ERK (Soeda et al., 2006, 2008). In our model we included the necessity

\section{REFERENCES}

Adamski, D., Mayol, J.-F., Platet, N., Berger, F., Hérodin, F., and Wion, D. (2007). Effects of Hoechst 33342 on $\mathrm{C} 2 \mathrm{c} 12$ and PC12 cell differentiation. FEBS Lett. 581, 3076-3080. doi: 10.1016/j.febslet.2007. 05.073

Adler, V., Qu, Y., Smith, S. J., Izotova, L., Pestka, S., Kung, H. F., et al. (2005). Functional interactions of Raf and MEK with Jun-N-terminal kinase (JNK) result in a positive feedback loop on the oncogenic Ras signaling pathway. Biochemistry 44, 10784-10795. doi: 10.1021/ bi050619j of UPAR signaling though the activation of late genes, such as $K l f 5$, yet the causal relationship between uPAR signaling and late gene expression remains unclear. However, uPAR signaling could constitute the additional positive feedbacks beyond MAPK signaling that were predicted by Ryu et al. (2015), which would be interesting to test on the single cell level. Reporters for UPAR and/or JNK activity should likewise show a heterogenous activity and correlate with the per-cell differentiation status, which could potentially be modeled within a stochastic differential equation framework.

In conclusion, our approach has identified the short and long-term transcriptional activity in PC12 cells after NGF and EGF stimulation. Modeling the pathway orchestration using a Boolean model we identified feedback regulations beyond MAPK signaling that attenuate and sustain the cellular decision toward differentiation. Extending on previous studies we established JNK as a key player in PC12 cell differentiation that might have equal, if not even more importance than ERK during this process. Over time AP1 was accompanied by a variety of transcription factors serving signal attenuation, signal maintenance and morphological change of the cell, which demonstrates that the decision toward differentiation is a time sequential process over at least $12 \mathrm{~h}$.

\section{AUTHOR CONTRIBUTIONS}

MF, SKn, MK, SK, and BO performed the experiments. MF, SKn, AS, and BO performed the data analysis. $\mathrm{HB}$ And $\mathrm{MB}$ conceived the project, performed the data analysis and wrote the manuscript with BO. All authors approved the final manuscript.

\section{ACKNOWLEDGMENTS}

This work was was supported by the Deutsche Forschungsgemeinschaft grant InKoMBio: SPP 1395. The authors greatly acknowledge the Genomics and Proteomics Core Facility, German Cancer Research Center/DKFZ, Heidelberg, Germany for their microarray service.

\section{SUPPLEMENTARY MATERIAL}

The Supplementary Material for this article can be found online at: http://journal.frontiersin.org/article/10.3389/fgene. 2016.00044 
Avraham, R., and Yarden, Y. (2011). Feedback regulation of EGFR signalling: decision making by early and delayed loops. Nat. Rev. Mol. Cell Biol. 12, 104-117. doi: 10.1038/nrm3048

Azzalini, A. (2015). The R package sn: The skew-normal and skew-t distributions (version 1.2-4). Universitate di Padova.

Bar-Joseph, Z., Gitter, A., and Simon, I. (2012). Studying and modelling dynamic biological processes using time-series gene expression data. Nat. Rev. Genet. 13, 552-564. doi: 10.1038/nrg3244

Bastide, P., Darido, C., Pannequin, J., Kist, R., Robine, S., Marty-Double, C., et al. (2007). Sox9 regulates cell proliferation and is required for Paneth cell differentiation in the intestinal epithelium. J. Cell Biol. 178, 635-648. doi: $10.1083 /$ jcb. 200704152

Ben-Dor, A., Shamir, R., and Yakhini, Z. (1999). Clustering gene expression patterns. J. Comput. Biol. 6, 281-297. doi: 10.1089/106652799318274

Burstein, D. E., Blumberg, P. M., and Greene, L. A. (1982). Nerve growth factorinduced neuronal differentiation of PC12 pheochromocytoma cells: lack of inhibition by a tumor promoter. Brain Res. 247, 115-119. doi: 10.1016/00068993(82)91033-2

Cao, X. M., Koski, R. A., Gashler, A., McKiernan, M., Morris, C. F., Gaffney, R., et al. (1990). Identification and characterization of the Egr-1 gene product, a DNA-binding zinc finger protein induced by differentiation and growth signals. Mol. Cell. Biol. 10, 1931-1939. doi: 10.1128/MCB.10.5.1931

Chao, M. V. (1992). Neurotrophin receptors: a window into neuronal differentiation. Neuron 9, 583-593. doi: 10.1016/0896-6273(92)90023-7

Chen, J.-Y., Lin, J.-R., Cimprich, K. A., and Meyer, T. (2012). A two-dimensional ERK-AKT signaling code for an NGF-triggered cell-fate decision. Mol. Cell 45, 196-209. doi: 10.1016/j.molcel.2011.11.023

Chung, J., Kubota, H., Ozaki, Y.-I., Uda, S., and Kuroda, S. (2010). Timingdependent actions of NGF required for cell differentiation. PLoS ONE 5:e9011. doi: 10.1371 /journal.pone.0009011

Chung, J., Miura, N., Ito, A., Sawada, M., Nishikawa, S., Kuroda, K., et al. (2014). Single-cell heterogeneity in suppression of PC12 differentiation by direct microinjection of a differentiation inhibitor, U0126. Cell Biol. Int. 38, 1215-1220. doi: 10.1002/cbin.10296

Cowley, S., Paterson, H., Kemp, P., and Marshall, C. J. (1994). Activation of MAP kinase kinase is necessary and sufficient for PC12 differentiation and for transformation of NIH 3T3 cells. Cell 77, 841-852. doi: 10.1016/00928674(94)90133-3

Dijkmans, T. F., van Hooijdonk, L. W. A., Schouten, T. G., Kamphorst, J. T., Fitzsimons, C. P., and Vreugdenhil, E. (2009). Identification of new Nerve Growth Factor-responsive immediate-early genes. Brain Res. 1249, 19-33. doi: 10.1016/j.brainres.2008.10.050

Dijkmans, T. F., van Hooijdonk, L. W. A., Schouten, T. G., Kamphorst, J. T., Vellinga, A. C. A., Meerman, J. H. N., et al. (2008). Temporal and functional dynamics of the transcriptome during nerve growth factorinduced differentiation. J. Neurochem. 105, 2388-2403. doi: 10.1111/j.14714159.2008.05338.x

Dikic, I., Schlessinger, J., and Lax, I. (1994). PC12 cells overexpressing the insulin receptor undergo insulin-dependent neuronal differentiation. Curr. Biol. 4, 702-708. doi: 10.1016/S0960-9822(00)00155-X

Eriksson, M., Taskinen, M., and Leppä, S. (2007). Mitogen activated protein kinasedependent activation of c-Jun and c-Fos is required for neuronal differentiation but not for growth and stress response in PC12 cells. J. Cell. Physiol. 210, 538-548. doi: 10.1002/jcp.20907

Farias-Eisner, R., Vician, L., Reddy, S., Basconcillo, R., Rabbani, S. A., Wu, Y. Y., et al. (2001). Expression of the urokinase plasminogen activator receptor is transiently required during "priming" of PC12 cells in nerve growth factordirected cellular differentiation. J. Neurosci. Res. 63, 341-346.

Farias-Eisner, R., Vician, L., Silver, A., Reddy, S., Rabbani, S. A., and Herschman, H. R. (2000). The urokinase plasminogen activator receptor (UPAR) is preferentially induced by nerve growth factor in $\mathrm{PC} 12$ pheochromocytoma cells and is required for NGF-driven differentiation. J. Neurosci. 20, 230-239.

Fey, D., Croucher, D. R., Kolch, W., and Kholodenko, B. N. (2012). Crosstalk and signaling switches in mitogen-activated protein kinase cascades. Front. Physiol. 3:355. doi: 10.3389/fphys.2012.00355

Fiore, M., Chaldakov, G. N., and Aloe, L. (2009). Nerve growth factor as a signaling molecule for nerve cells and also for the neuroendocrine-immune systems. Rev. Neurosci. 20, 133-145. doi: 10.1515/REVNEURO.2009.20.2.133
Fontanet, P., Irala, D., Alsina, F. C., Paratcha, G., and Ledda, F. (2013). Pea3 transcription factor family members Etv4 and Etv5 mediate retrograde signaling and axonal growth of DRG sensory neurons in response to NGF. J. Neurosci. 33, 15940-15951. doi: 10.1523/JNEUROSCI.0928-13.2013

Gil, G. A., Bussolino, D. F., Portal, M. M., Alfonso Pecchio, A., Renner, M. L., Borioli, G. A., et al. (2004). c-Fos activated phospholipid synthesis is required for neurite elongation in differentiating PC12 cells. Mol. Biol. Cell 15, 18811894. doi: 10.1091/mbc.E03-09-0705

Giraldo, A., Barrett, O. P. T., Tindall, M. J., Fuller, S. J., Amirak, E., Bhattacharya, B. S., et al. (2012). Feedback regulation by Atf3 in the endothelin-1-responsive transcriptome of cardiomyocytes: Egr1 is a principal Atf3 target. Biochem. J. 444, 343-355. doi: 10.1042/BJ20120125

Gotoh, Y., Nishida, E., Yamashita, T., Hoshi, M., Kawakami, M., and Sakai, H. (1990). Microtubule-associated-protein (MAP) kinase activated by nerve growth factor and epidermal growth factor in PC12 cells. Identity with the mitogen-activated MAP kinase of fibroblastic cells. Eur. J. Biochem. 193, 661669. doi: 10.1111/j.1432-1033.1990.tb19384.x

Greene, L. A., and Tischler, A. S. (1976). Establishment of a noradrenergic clonal line of rat adrenal pheochromocytoma cells which respond to nerve growth factor. Proc. Natl. Acad. Sci. U.S.A 73, 2424-2428. doi: 10.1073/pnas.73.7.2424

Györy, I., Boller, S., Nechanitzky, R., Mandel, E., Pott, S., Liu, E., et al. (2012). Transcription factor Ebf1 regulates differentiation stage-specific signaling, proliferation, and survival of B cells. Genes Dev. 26, 668-682. doi: 10.1101/gad.187328.112

Hallstrom, T. C., Mori, S., and Nevins, J. R. (2008). An E2f1-dependent gene expression program that determines the balance between proliferation and cell death. Cancer Cell 13, 11-22. doi: 10.1016/j.ccr.2007.11.031

Hoffmann, E., Thiefes, A., Buhrow, D., Dittrich-Breiholz, O., Schneider, H., Resch, K., et al. (2005). MEK1-dependent delayed expression of Fos-related antigen1 counteracts c-Fos and p65 NF-kappaB-mediated interleukin- 8 transcription in response to cytokines or growth factors. J. Biol. Chem. 280, 9706-9718. doi: 10.1074/jbc.M407071200

Huang, L., Feng, G., Du, P., Xia, T., Wang, X., Jing, W., et al. (2014). GeneAnswers: Integrated Interpretation of Genes. R package version 2.10.0.

Ito, E., Sweterlitsch, L. A., Tran, P. B., Rauscher, F. J. III, and Narayanan, R. (1990). Inhibition of PC-12 cell differentiation by the immediate early gene fra-1. Oncogene 5, 1755-1760.

Jing, S., Tapley, P., and Barbacid, M. (1992). Nerve growth factor mediates signal transduction through trk homodimer receptors. Neuron 9, 1067-1079. doi: 10.1016/0896-6273(92)90066-M

Johnson, W. E., Li, C., and Rabinovic, A. (2007). Adjusting batch effects in microarray expression data using empirical Bayes methods. Biostat 8, 118-127. doi: 10.1093/biostatistics/kxj037

Kandemir, B., Caglayan, B., Hausott, B., Erdogan, B., Dag, U., Demir, O., et al. (2014). Pea3 transcription factor promotes neurite outgrowth. Front. Mol. Neurosci. 7:59. doi: 10.3389/fnmol.2014.00059

Kannan, M. B., Solovieva, V., and Blank, V. (2012). The small MAF transcription factors MAFF, MAFG and MAFK: current knowledge and perspectives. Biochim. Biophys. Acta 1823, 1841-1846. doi: 10.1016/j.bbamcr.2012. 06.012

Kaplan, D. R., Martin-Zanca, D., and Parada, L. F. (1991). Tyrosine phosphorylation and tyrosine kinase activity of the trk proto-oncogene product induced by NGF. Nature 350, 158-160. doi: 10.1038/350158a0

Klesse, L. J., Meyers, K. A., Marshall, C. J., and Parada, L. F. (1999). Nerve growth factor induces survival and differentiation through two distinct signaling cascades in PC12 cells. Oncogene 18, 2055-2068. doi: 10.1038/sj.onc.1202524

Kueh, H. Y., Champhekar, A., Champhekhar, A., Nutt, S. L., Elowitz, M. B., and Rothenberg, E. V. (2013). Positive feedback between PU.1 and the cell cycle controls myeloid differentiation. Science 341, 670-673. doi: $10.1126 /$ science. 1240831

Lee, K.-H., Ryu, C. J., Hong, H. J., Kim, J., and Lee, E. H. (2005). CDNA microarray analysis of nerve growth factor-regulated gene expression profile in rat $\mathrm{PC} 12$ cells. Neurochem. Res. 30, 533-540. doi: 10.1007/s11064-005-2688-y

Leppä, S., Saffrich, R., Ansorge, W., and Bohmann, D. (1998). Differential regulation of c-Jun by ERK and JNK during PC12 cell differentiation. EMBO J. 17, 4404-4413. doi: 10.1093/emboj/17.15.4404

Levi-Montalcini, R. (1987). The nerve growth factor 35 years later. Science 237, 1154-1162. doi: 10.1126/science.3306916 
Levkovitz, Y., and Baraban, J. M. (2002). A dominant negative Egr inhibitor blocks nerve growth factor-induced neurite outgrowth by suppressing c-Jun activation: role of an Egr/c-Jun complex. J. Neurosci. 22, 3845-3854.

Levkovitz, Y., O'Donovan, K. J., and Baraban, J. M. (2001). Blockade of NGFinduced neurite outgrowth by a dominant-negative inhibitor of the egr family of transcription regulatory factors. J. Neurosci. 21, 45-52.

Luo, J., West, J. R., Cook, R. T., and Pantazis, N. J. (1999). Ethanol induces cell death and cell cycle delay in cultures of pheochromocytoma PC12 cells. Alcohol. Clin. Exp. Res. 23, 644-656. doi: 10.1111/j.1530-0277.1999.tb0 4166.x

Luo, W., Friedman, M. S., Shedden, K., Hankenson, K. D., and Woolf, P. J. (2009). GAGE: generally applicable gene set enrichment for pathway analysis. $B M C$ Bioinformatics 10:161. doi: 10.1186/1471-2105-10-161

Marek, L., Levresse, V., Amura, C., Zentrich, E., Van Putten, V., Nemenoff, R. A., et al. (2004). Multiple signaling conduits regulate global differentiationspecific gene expression in PC12 cells. J. Cell. Physiol. 201, 459-469. doi: $10.1002 /$ jcp. 20087

Marshall, C. J. (1995). Specificity of receptor tyrosine kinase signaling: transient versus sustained extracellular signal-regulated kinase activation. Cell 80, 179185. doi: 10.1016/0092-8674(95)90401-8

Mitrophanov, A. Y., and Groisman, E. A. (2008). Positive feedback in cellular control systems. Bioessays 30, 542-555. doi: 10.1002/bies.20769

Mullenbrock, S., Shah, J., and Cooper, G. M. (2011). Global expression analysis identified a preferentially nerve growth factor-induced transcriptional program regulated by sustained mitogen-activated protein kinase/extracellular signalregulated kinase (ERK) and AP-1 protein activation during PC12 cell differentiation. J. Biol. Chem. 286, 45131-45145. doi: 10.1074/jbc.M111. 274076

Murphy, L. O., and Blenis, J. (2006). MAPK signal specificity: the right place at the right time. Trends Biochem. Sci. 31, 268-275. doi: 10.1016/j.tibs.2006.03.009

Murphy, L. O., MacKeigan, J. P., and Blenis, J. (2004). A network of immediate early gene products propagates subtle differences in mitogen-activated protein kinase signal amplitude and duration. Mol. Cell. Biol. 24, 144-153. doi: 10.1128/MCB.24.1.144-153.2004

Murphy, L. O., Smith, S., Chen, R.-H., Fingar, D. C., and Blenis, J. (2002). Molecular interpretation of ERK signal duration by immediate early gene products. Nat. Cell Biol. 4, 556-564. doi: 10.1038/ncb822

Müssel, C., Hopfensitz, M., and Kestler, H. A. (2010). BoolNet-an R package for generation, reconstruction and analysis of Boolean networks. Bioinformatics 26, 1378-1380. doi: 10.1093/bioinformatics/btq124

Pang, L., Sawada, T., Decker, S. J., and Saltiel, A. R. (1995). Inhibition of MAP kinase kinase blocks the differentiation of PC-12 cells induced by nerve growth factor. J. Biol. Chem. 270, 13585-13588. doi: 10.1074/jbc.270. 23.13585

Pons, J., Kitlinska, J., Jacques, D., Perreault, C., Nader, M., Everhart, L., et al. (2008). Interactions of multiple signaling pathways in neuropeptide $\mathrm{Y}$-mediated bimodal vascular smooth muscle cell growth. Can. J. Physiol. Pharmacol. 86, 438-448. doi: 10.1139/Y08-054

Qui, M. S., and Green, S. H. (1992). PC12 cell neuronal differentiation is associated with prolonged p 21 ras activity and consequent prolonged ERK activity. Neuron 9, 705-717. doi: 10.1016/0896-6273(92)90033-A

Ritchie, M. E., Dunning, M. J., Smith, M. L., Shi, W., and Lynch, A. G. (2011). BeadArray expression analysis using bioconductor. PLoS Comput. Biol. 7:e1002276. doi: 10.1371/journal.pcbi.1002276

Ryu, H., Chung, M., Dobrzynski, M., Fey, D., Blum, Y., Lee, S. S., et al. (2015). Frequency modulation of ERK activation dynamics rewires cell fate. Mol. Syst. Biol. 11, 838-838. doi: 10.15252/msb.20156458

Saez-Rodriguez, J., Alexopoulos, L. G., Epperlein, J., Samaga, R., Lauffenburger, D. A., Klamt, S., et al. (2009). Discrete logic modelling as a means to link protein signalling networks with functional analysis of mammalian signal transduction. Mol. Syst. Biol. 5:331. doi: 10.1038/msb.2009.87

Saito, T. H., Uda, S., Tsuchiya, T., Ozaki, Y.-I., and Kuroda, S. (2013). Temporal decoding of MAP kinase and CREB phosphorylation by selective immediate early gene expression. PLoS ONE 8:e57037. doi: 10.1371/journal.pone.00 57037

Santos, S. D., Verveer, P. J., and Bastiaens, P. I. (2007). Growth factor-induced MAPK network topology shapes Erk response determining PC-12 cell fate. Nat. Cell Biol. 9, 324-330. doi: 10.1038/ncb1543
Sasagawa, S., Ozaki, Y., Fujita, K., and Kuroda, S. (2005). Prediction and validation of the distinct dynamics of transient and sustained ERK activation. Nat. Cell Biol. 7, 365-373. doi: 10.1038/ncb1233

Selbie, L. A., and Hill, S. J. (1998). G protein-coupled-receptor cross-talk: the finetuning of multiple receptor-signalling pathways. Trends Pharmacol. Sci. 19, 87-93. doi: 10.1016/S0165-6147(97)01166-8

Shim, K. S., Rosner, M., Freilinger, A., Lubec, G., and Hengstschläger, M. (2006). Bach2 is involved in neuronal differentiation of N1E-115 neuroblastoma cells. Exp. Cell Res. 312, 2264-2278. doi: 10.1016/j.yexcr.2006.03.018

Singh, A., Nascimento, J. M., Kowar, S., Busch, H., and Boerries, M. (2012). Boolean approach to signalling pathway modelling in HGFinduced keratinocyte migration. Bioinformatics 28, i495-i501. doi: 10.1093/bioinformatics/bts410

Soeda, S., Koyanagi, S., Kuramoto, Y., Kimura, M., Oda, M., Kozako, T., et al. (2008). Anti-apoptotic roles of plasminogen activator inhibitor-1 as a neurotrophic factor in the central nervous system. Thromb. Haemost. 100, 1014-1020. doi: 10.1160/th08-04-0259

Soeda, S., Shinomiya, K., Ochiai, T., Koyanagi, S., Toda, A., Eyanagi, R., et al. (2006). Plasminogen activator inhibitor-1 aids nerve growth factor-induced differentiation and survival of pheochromocytoma cells by activating both the extracellular signal-regulated kinase and c-Jun pathways. Neuroscience 141, 101-108. doi: 10.1016/j.neuroscience.2006.03.026

Sparta, B., Pargett, M., Minguet, M., Distor, K., Bell, G., and Albeck, J. G. (2015). Receptor level mechanisms are required for epidermal growth factor (EGF)stimulated extracellular signal-regulated kinase (ERK) activity pulses. J. Biol. Chem. 290, 24784-24792. doi: 10.1074/jbc.M115.662247

Strickert, M., Teichmann, S., Sreenivasulu, N., and Seiffert, U. (2005). "Highthroughput multi-dimensional scaling (HiT-MDS) for cDNA-array expression data," in Artificial Neural Networks: Biological Inspirations ICANN 2005, number 3696 in Lecture Notes in Computer Science, eds W. Duch, J. Kacprzyk, E. Oja, and S. A. Zadrrony (Berlin; Heidelberg: Springer), 625-633.

Subramanian, A., Tamayo, P., Mootha, V. K., Mukherjee, S., Ebert, B. L., Gillette, M. A., et al. (2005). Gene set enrichment analysis: a knowledge-based approach for interpreting genome-wide expression profiles. Proc. Natl. Acad. Sci. U.S.A. 102, 15545-15550. doi: 10.1073/pnas.0506580102

Tanabe, K., Bonilla, I., Winkles, J. A., and Strittmatter, S. M. (2003). Fibroblast growth factor-inducible-14 is induced in axotomized neurons and promotes neurite outgrowth. J. Neurosci. 23, 9675-9686.

Tiedje, C., Ronkina, N., Tehrani, M., Dhamija, S., Laass, K., Holtmann, H., et al. (2012). The p38/MK2-driven exchange between tristetraprolin and HuR regulates AURich elementdependent translation. PLoS Genet. 8:e1002977. doi: 10.1371/journal.pgen.1002977

Tirone, F. (2001). The gene PC3(TIS21/BTG2), prototype member of the PC3/BTG/TOB family: regulator in control of cell growth, differentiation, and DNA repair? J. Cell. Physiol. 187, 155-165. doi: 10.1002/jcp.1062

Vaudry, D., Stork, P. J., Lazarovici, P., and Eiden, L. E. (2002). Signaling pathways for PC12 cell differentiation: making the right connections. Science 296, 16481649. doi: $10.1126 /$ science. 1071552

Vician, L., Basconcillo, R., and Herschman, H. R. (1997). Identification of genes preferentially induced by nerve growth factor versus epidermal growth factor in PC12 pheochromocytoma cells by means of representational difference analysis. J. Neurosci. Res. 50, 32-43.

von Kriegsheim, A., Baiocchi, D., Birtwistle, M., Sumpton, D., Bienvenut, W., Morrice, N., et al. (2009). Cell fate decisions are specified by the dynamic ERK interactome. Nat. Cell Biol. 11, 1458-1464. doi: 10.1038/ncb1994

Waetzig, V., and Herdegen, T. (2003). The concerted signaling of ERK1/2 and JNKs is essential for PC12 cell neuritogenesis and converges at the level of target proteins. Mol. Cell. Neurosci. 24, 238-249. doi: 10.1016/S1044-7431(03) 00126-X

Weber, S., Fernandez-Cachon, M. L., Nascimento, J. M., Knauer, S., Offermann, B., Murphy, R. F., et al. (2013). Label-free detection of neuronal differentiation in cell populations using high-throughput live-cell imaging of PC12 cells. PLoS ONE 8:e56690. doi: 10.1371/journal.pone.0056690

$\mathrm{Wu}$, Y. Y., and Bradshaw, R. A. (1996). Synergistic induction of neurite outgrowth by nerve growth factor or epidermal growth factor and interleukin-6 in PC12 cells. J. Biol. Chem. 271, 13033-13039. doi: 10.1074/jbc.271.22.13033

Xing, J., Kornhauser, J. M., Xia, Z., Thiele, E. A., and Greenberg, M. E. (1998). Nerve growth factor activates extracellular signal-regulated kinase and 
p38 mitogen-activated protein kinase pathways to stimulate CREB serine 133 phosphorylation. Mol. Cell. Biol. 18, 1946-1955. doi: 10.1128/MCB.18. 4.1946

Xiong, W., and Ferrell, J. E. (2003). A positive-feedback-based bistable 'memory module' that governs a cell fate decision. Nature 426, 460-465. doi: 10.1038 /nature02089

Yoon, H. S., Chen, X., and Yang, V. W. (2003). Krüppel-like factor 4 mediates p53dependent G1/S cell cycle arrest in response to DNA damage. J. Biol. Chem. 278, 2101-2105. doi: 10.1074/jbc.M211027200

Yosef, N., and Regev, A. (2011). Impulse control: temporal dynamics in gene transcription. Cell 144, 886-896. doi: 10.1016/j.cell.2011. 02.015

Zhang, J.-P., Zhang, H., Wang, H.-B., Li, Y.-X., Liu, G.-H., Xing, S., et al. (2014). Down-regulation of Sp1 suppresses cell proliferation, clonogenicity and the expressions of stem cell markers in nasopharyngeal carcinoma. J. Trans. Med. 12, 222. doi: 10.1186/s12967-014-0222-1

Conflict of Interest Statement: The authors declare that the research was conducted in the absence of any commercial or financial relationships that could be construed as a potential conflict of interest.

Copyright (c) 2016 Offermann, Knauer, Singh, Fernández-Cachón, Klose, Kowar, Busch and Boerries. This is an open-access article distributed under the terms of the Creative Commons Attribution License (CC BY). The use, distribution or reproduction in other forums is permitted, provided the original author (s) or licensor are credited and that the original publication in this journal is cited, in accordance with accepted academic practice. No use, distribution or reproduction is permitted which does not comply with these terms. 